\title{
Sistema sensor com câmera USB para uso em experimentos de polarização
} da $l u z^{+*}$

\author{
José Luís Fabris ${ }^{1}$ \\ Marcia Muller ${ }^{2}$ \\ Universidade Tecnológica Federal do Paraná \\ Luís Victor Muller Fabris ${ }^{3}$ \\ Estudante de Física - Universidade Federal do Paraná \\ Curitiba - PR
}

\section{Resumo}

Este trabalho apresenta um sistema sensor para uso em laboratórios de ensino, composto por uma câmera USB e um software desenvolvido e disponibilizado pelos autores. O sistema sensor é adequado para o estudo de fenômenos associados à propriedade de polarização da luz, tendo sido testado em experimentos realizados para verificar a Lei de Malus e a eficiência espectral de polarizadores. São apresentados detalhes da montagem experimental, na qual a luz de uma lanterna de LED usada como fonte luminosa no visível é projetada numa tela branca após passar por dois polarizadores. A imagem projetada na tela é captada pela câmera e o software fornece a intensidade luminosa relativa da luz. Com o uso de dois polarizadores lineares tipo H rotatórios, ajustes lineares da Lei de Malus aos dados de intensidade da luz transmitida apresentaram coeficientes de correlação $R$ maiores do que 0,9988. A eficiência dos polarizadores em diferentes regiões do espectro eletromagnético visível foi analisada com o auxílio de filtros de cor inseridos à montagem experimental. O sistema também foi empregado na avaliação da estabilidade temporal da intensidade do LED de luz branca.

\footnotetext{
${ }^{+}$Sensing system with USB camera for experiments of polarization of the light

* Recebido: novembro de 2016.

Aceito: maio de 2017.

1 E-mail: fabrisj1@gmail.com

2 E-mail: marcia.muller.br@gmail.com

3 E-mail: luis.victor.m@gmail.com
} 
Palavras-chave: Detecção de luz; Polarização; Lei de Malus.

\begin{abstract}
This work shows a sensor system for educational experiments, composed of a USB camera and a software developed and provided by the authors. The sensor system is suitable for the purpose of studying phenomena related to the polarization of the light. The system was tested in experiments performed to verify the Malus' Law and the spectral efficiency of polarizers. Details of the experimental setup are shown. The camera captures the light in the visible spectral range from a LED that illuminates a white screen after passing through two polarizers. The software uses the image captured by the camera to provide the relative intensity of the light. With the use of two rotating $H$-sheet linear polarizers, a linear fitting of the Malus's Law to the transmitted light intensity data resulted in correlation coefficients $R$ larger than 0.9988. The efficiency of the polarizers in different visible spectral regions was verified with the aid of color filters added to the experimental setup. The system was also used to evaluate the intensity time stability of a white LED.
\end{abstract}

Keywords: Light detection; Polarization; Malus' Law.

\title{
I. Introdução
}

Interferência, difração e polarização, são fenômenos fundamentais abordados no estudo da Teoria Ondulatória da Luz. Paralelamente a uma abordagem teórica dos fenômenos físicos desta Teoria, aulas experimentais se constituem em uma ferramenta poderosa para facilitar a compreensão e motivar os estudantes. Experimentos quantitativos envolvendo interferência e difração são facilmente realizáveis com materiais e equipamentos de baixo custo, como fendas e fontes de luz (LOPES, 2004; CATELLI, 2004). No entanto, experimentos quantitativos para o estudo da polarização da luz, como o da verificação da lei de Malus, requerem equipamentos que realizem a medição da intensidade luminosa. Equipamentos comerciais para isto tem um custo elevado, inviabilizando a realização de tais experimentos em instituições de ensino que não tenham condições de adquiri-los.

A Lei de Malus, publicada originalmente em 1809 por Étienne Malus, estabelece que se uma onda luminosa linearmente polarizada (ou plano-polarizada, também chamada de estado- $P$ ) incidir sobre um polarizador linear ideal (um analisador) com seu vetor campo elétrico $\boldsymbol{E}$ formando um ângulo $\theta$ com a direção de transmissão do analisador, a intensidade $I$ da luz transmitida será proporcional ao cosseno ao quadrado do ângulo $\theta$ (HECHT, 1987), como na equação (1). 


$$
I=I_{0} \cos ^{2} \theta
$$

Alguns trabalhos previamente publicados se focam na questão da viabilização de experimentos, propondo estratégias alternativas de baixo custo para a leitura da intensidade luminosa. $\mathrm{O}$ fotodetector de um telefone celular, normalmente utilizado para controlar a luminosidade da tela, foi usado para a leitura da intensidade da luz fazendo uso de aplicativos adicionais (VIEIRA, 2015). No entanto, os softwares disponíveis para realizar esta medição não permitem a exportação dos dados para análise e o smartphone não é um equipamento de baixo custo que seja normalmente encontrado em laboratórios e que, portanto, possa ser emprestado para alunos que não o possuam. Outro trabalho propõe o uso de uma câmera fotográfica digital (VERTCHENKO, 2016) que apresente a possibilidade de desabilitar o ajuste automático de exposição, uma funcionalidade que normalmente não está disponível nas câmeras mais baratas que são encontradas no mercado. Um outro trabalho (PEDROSO, 2016) ainda propõe a fabricação de um luxímetro usando um fotodiodo e um multímetro, mas tal estratégia necessita de uma calibração prévia pois a resposta do fotodiodo não é linear.

Neste trabalho são apresentados resultados da aplicação de um sistema sensor, desenvolvido especificamente para uso em laboratórios de ensino, num experimento para a verificação da Lei de Malus. O sistema possibilita a medição de intensidade luminosa relativa utilizando uma câmera USB que pode ser adquirida com custo baixo. Alternativamente pode também ser utilizada a câmera que já vem instalada na maioria dos laptops. O software desenvolvido para este propósito realiza a compensação do ajuste automático de exposição da câmera no momento da medição, mostrando o resultado já corrigido, evitando assim a necessidade do uso de uma câmera com ajuste manual de exposição, bem como de calibração prévia. Também é possível salvar os dados experimentais de medições realizadas em intervalos de tempo regulares indicados pelo usuário, possibilitando um posterior tratamento estatístico de incertezas para compor as barras de erro dos gráficos.

\section{Metodologia}

\section{II.A Software do sistema sensor}

O software Power meter desenvolvido neste trabalho pode ser obtido a partir do endereço <http://powermeter.luisvmf.com> nas versões para Linux® ou Windows®**. O programa foi construído com o Electron $($ (GITHUB INC, 2016) e calcula a intensidade luminosa relativa com base na imagem capturada pela câmera. A aquisição da imagem da câmera é feita por meio do método getUserMedia. A imagem é desenhada em um elemento video e em seguida copiada para um canvas. Uma função executada por um temporizador a cada aproximadamente 50 ms determina as componentes RGB (Red, Green, Blue) médias na área selecionada pelo usuário e que é iluminada pela fonte (área da medida), bem como em uma área

\footnotetext{
** Linux ${ }^{\circledR}$ is the registered trademark of Linus Torvalds in the U.S. and other countries. Windows is a registered trademark of Microsoft Corporation in the United States and/or other countries.
} 
pré-definida e fixa que recebe somente luz ambiente de intensidade constante que irá servir como referência (área da referência). As componentes RGB são representadas por três números inteiros que variam de 0 a 255 e que tem uma relação com a intensidade das componentes vermelha, verde e azul de um pixel. A determinação das componentes RGB médias é realizada pelo método getImageData da API Canvas 2D. Em seguida o programa calcula a intensidade luminosa relativa $I$ (em unidades arbitrárias) que é capturada pela câmera, de acordo com a equação (2):

$$
I=\frac{A\left(R_{m e d}+G_{m e d}+B_{m e d}\right)}{\left(R_{r e f}+G_{r e f}+B_{r e f}\right)}
$$

Na equação (2), $R_{r e f}, G_{r e f}$ e $B_{r e f}$ são as componentes RGB médias na área da referência e $R_{m e d}, G_{m e d}$ e $B_{m e d}$ são as componentes RGB médias na área da medida. A constante $A$ (foi utilizado o valor $A=30$ ) define a amplitude de excursão do sinal. Como a intensidade luminosa na área de referência é mantida aproximadamente constante durante a realização do experimento, esta equação compensa variações da exposição automática da câmera, que ocorrem na maioria dos modelos.

Durante o experimento, deve ser tomado cuidado para evitar a saturação do detector com intensidades luminosas muito elevadas. Na prática, o programa realiza a medição das intensidades RGB de todos os pixels (área de referência mais área da medida), emitindo um alerta quando o número de pixels com intensidade acima de 253 excede 85. Além disso, é necessária uma intensidade luminosa mínima detectável pela câmera na área de referência para fazer a compensação da exposição. O programa emite um alerta sempre que a intensidade na área de referência é menor ou igual a $25 \%$ da intensidade na área da medida. Para facilitar a identificação da origem da eventual saturação de pixels, um gráfico da intensidade ao longo de uma linha horizontal de pixels da câmera é exibido na janela do programa. A posição y desta linha pode ser definida pelo usuário com o auxílio do seletor deslizante posicionado na parte inferior da janela do programa, e é indicada por uma linha verde sobre a imagem da câmera. Este gráfico é construído com a biblioteca Aristochart $\odot$ (COONEY, 2016). O valor da intensidade dado pela equação (2) é arredondado para a primeira casa decimal para ser exibido para o usuário na janela do programa. Um gráfico mostrando os últimos valores medidos de intensidade também é exibido na janela do programa. Para desenhar o gráfico é utilizada a biblioteca livre smoothie charts@ (WALNES, 2016).

O usuário pode gravar um determinado número de medições separadas por um intervalo de tempo escolhido utilizando os controles localizados na parte central da janela do programa. Os dados são gravados em um arquivo delimitado por tab utilizando o módulo filesystem que vem com o Electron $\odot$, com o tempo em milissegundos decorrido desde a primeira medição na primeira coluna e o valor da intensidade relativa na segunda coluna. Este tipo de arquivo pode ser importado facilmente em softwares para tratamento de dados e criação de gráficos como o SciDavis@ ou o Gnuplot $@$. 
Um exemplo típico da janela de aquisição do software é visto na Fig. 1. A área retangular de dimensões fixas a esquerda da imagem da câmera (canto superior esquerdo da tela) mostra a área iluminada somente com luz ambiente que é utilizada como referência para o cálculo da intensidade relativa. Os controles deslizáveis $x i, x f, y i$ e $y f$, permitem delimitar a área retangular da região iluminada capturada pela câmera que corresponde à área da medida usada no cálculo da intensidade relativa, cujo gráfico temporal é mostrado na região superior à direita da tela.

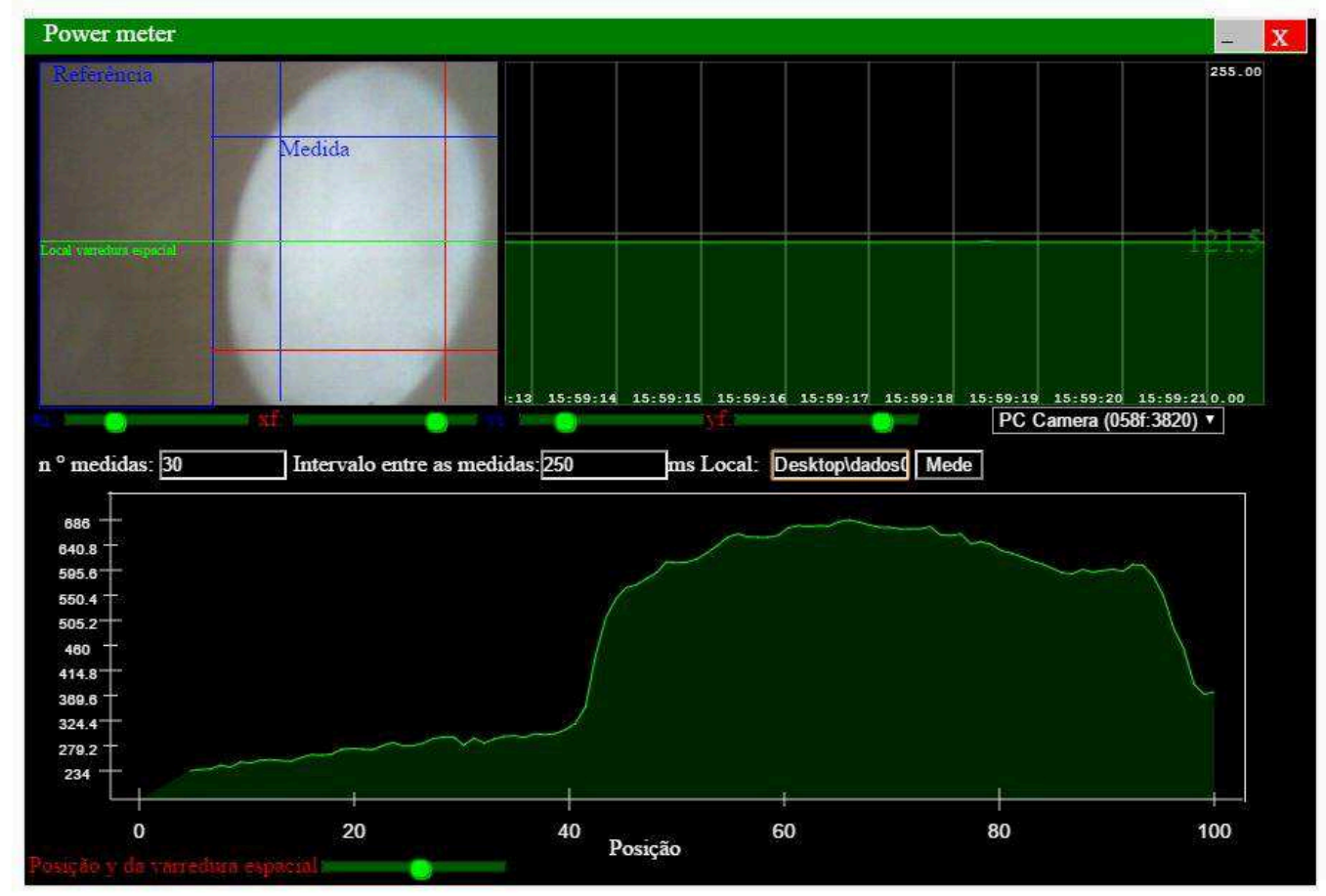

Fig. 1 - Imagem da janela do programa desenvolvido neste trabalho, usado para medir intensidade relativa da luz com a câmera.

Para permitir a gravação dos dados, também devem ser informados pelo usuário o número de medições a serem realizadas, o intervalo temporal entre estas medições, além do nome e local de destino do arquivo de dados. Na metade inferior da tela é mostrado o gráfico da intensidade da luz na linha de pixels definida pelo controle deslizante localizado abaixo deste gráfico. Esta intensidade é usada na visualização de eventuais saturações de pixels que possam ocorrer durante o experimento.

Para evitar oscilações indesejadas da intensidade relativa medida, a intensidade na região de referência deve permanecer constante durante todo o experimento. É necessário minimizar a incidência de luz do sol através de janelas, pois esta pode resultar em variações da intensidade ao longo do experimento. Deve-se tomar cuidado, pois se houver muita luz a câmera irá reduzir a exposição, gerando uma menor sensibilidade. No entanto, a área de referência deve receber iluminação adequada para garantir um nível detectável de iluminação. 
Uma vez definidas as condições do ambiente no qual o experimento será realizado, deve-se realizar uma varredura de toda a escala dinâmica da intensidade da luz a ser medida, observando-se possíveis avisos de saturação nas áreas de medição e referência, ou de baixa intensidade na área de referência.

\section{II.B Montagem experimental}

O sistema sensor foi testado com experimentos de polarização realizados para verificar a Lei de Malus e a eficiência espectral dos polarizadores. Para a realização dos experimentos foram utilizados dois polarizadores para luz visível, dois filtros coloridos (azul e vermelho), uma lanterna LED, e o sistema sensor composto por uma câmera USB e um computador além do software desenvolvido. Foi usado um banco ótico linear e suporte de polarizadores (EQ045, Cidepe) na montagem experimental; no entanto, esta pode ser realizada com outros sistemas ou mesmo com materiais alternativos.

Um dos polarizadores atua como polarizador propriamente dito, transformado a luz incidente não polarizada da fonte luminosa em luz com estado de polarização $P$, ou seja, luz linearmente polarizada com vetor campo elétrico na direção do eixo de polarização. O segundo polarizador atua como um analisador da luz incidente com estado de polarização $P$, deixando passar a componente do vetor campo elétrico na direção do eixo de polarização. Mudanças no ângulo entre os eixos de polarização do polarizador e do analisador alteram a amplitude do vetor campo elétrico transmitido e, consequentemente, a intensidade da luz de acordo com a Lei de Malus fornecida pela equação (1). Fotos da montagem experimental são mostradas na Fig. 2.

A luz passa pelos polarizadores posicionados nos suportes com ajuste de ângulo e é projetada em uma tela de papel branco. A câmera USB está voltada para o papel de modo que uma região somente com luz ambiente fique na área de referência do programa e a região iluminada pela luz da lanterna fique na área da medida. $\mathrm{O}$ ângulo entre os eixos de polarização dos dois polarizadores foi variado entre $0^{\circ}$ e $170^{\circ} \mathrm{em}$ passos de $10^{\circ}$ alterando a direção do eixo do analisador.

As diversas aquisições realizadas para cada ângulo permitiram um posterior tratamento estatístico dos dados, para determinação das barras de incerteza associadas às medições. No experimento descrito neste trabalho foi usado como sensor uma câmera marca C3 Tech com conexão USB. 

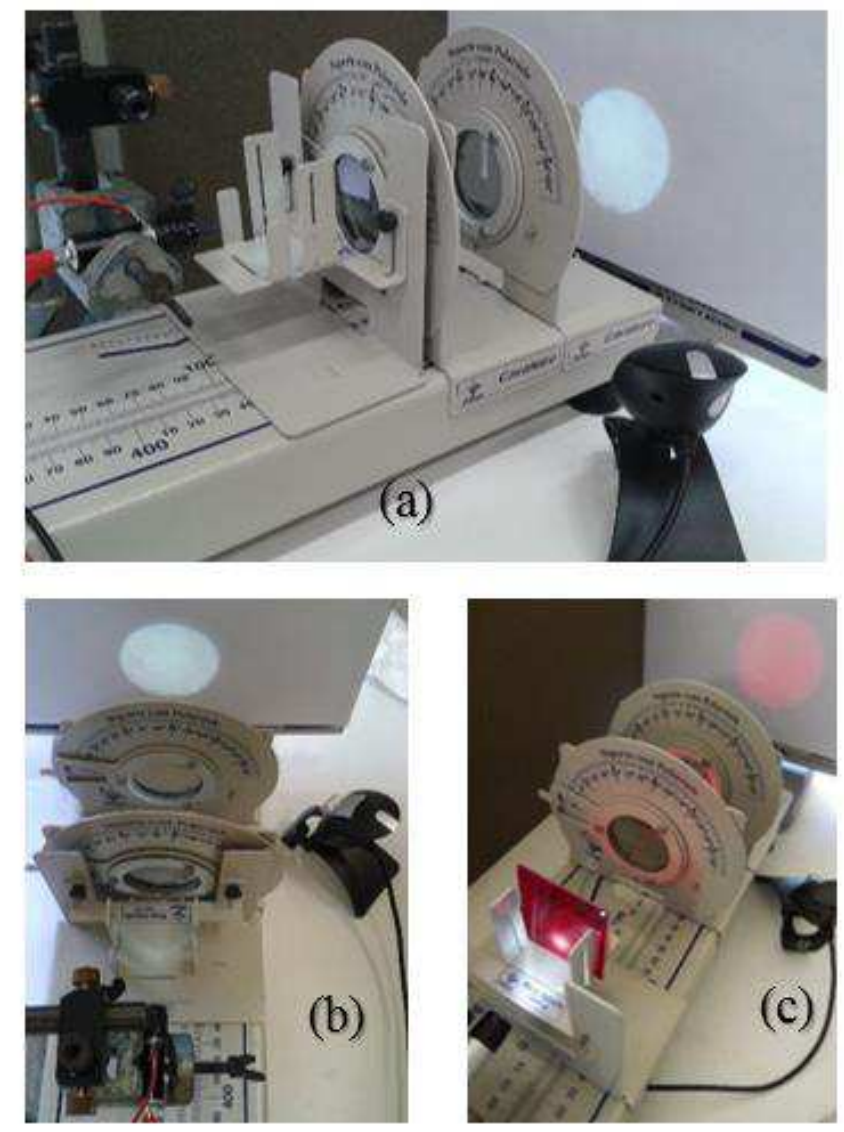

Fig. 2 - Foto da montagem experimental para a verificação da Lei de Malus: a) vista lateral; b) vista superior, c) com filtro colorido.

Na escolha dos polarizadores a opção mais acessível são os filmes polarizadores do tipo H, idealizados por E. H. Land em 1938 (LAND, 1951). Este tipo de polarizador, conhecido comercialmente como lâmina Polaroide $H$, apresenta uma razão de extinção (porcentagem de transmitância para $\theta=90^{\circ}$ na Lei de Malus) próxima de $10^{-5}$ para toda a faixa visível do espectro eletromagnético, com uma queda na eficiência na região do ultravioleta que resulta em razões de extinção da ordem de $10^{-2}$. Polarizadores tipo $\mathrm{H}$ também podem ser projetados para operar no infravermelho, mas com razões de extinção que não se comparam às dos polarizadores H no visível (RÄTY, 2004).

Como resultado combinado das eficiências dos polarizadores $\mathrm{H}$ para diferentes regiões do espectro, bem como da eficiência diferenciada da câmera em diferentes comprimentos de onda, uma fonte de luz adequada para o experimento é uma lanterna LED, com emissão predominantemente na região visível do espectro. Neste trabalho foi utilizada uma lanterna de LED com lente de focalização contendo um único LED emitindo luz branca.

Realizando o experimento da verificação da Lei de Malus com a luz da lanterna espectralmente filtrada por filtros de cor é possível verificar também a eficiência dos polarizadores em diferentes regiões espectrais do visível. Para analisar a resposta dos polarizadores a 
diferentes cores, foram posicionados filtros de cor após a lanterna conforme mostrado na Fig. 2c.

Na Fig. 3, encontra-se o espectro da irradiância espectral relativa da lanterna LED usada com e sem filtragem espectral.

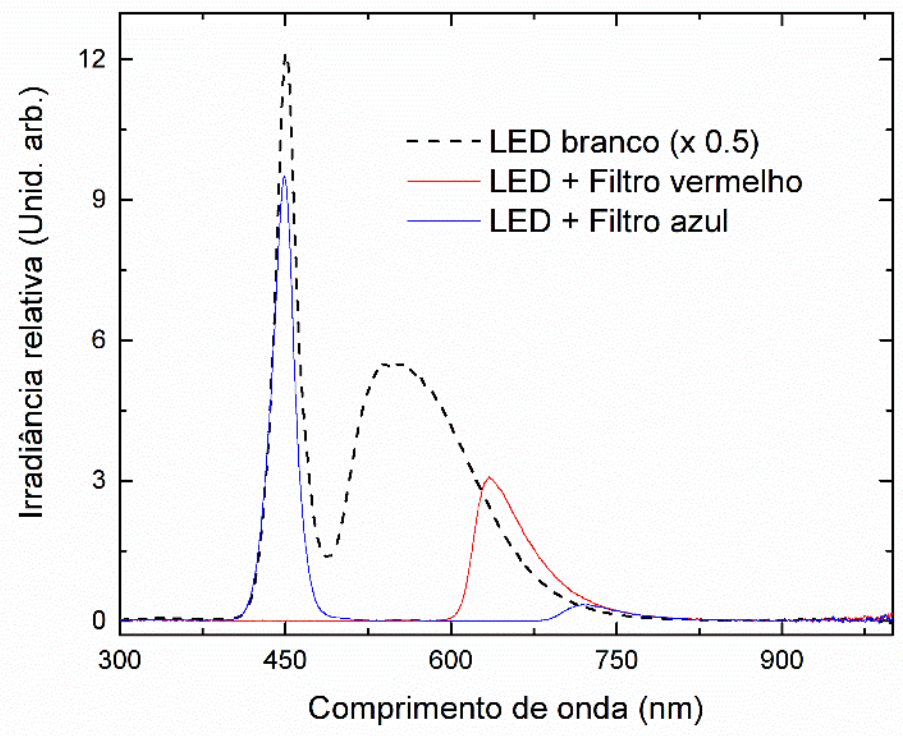

Fig. 3 - Espectro da irradiância espectral da lanterna LED empregada nos experimentos.

O espectro foi medido com um espectrômetro a fibra ótica (Ocean Optics, HR4000), com a resposta corrigida para a configuração experimental utilizada. A linha tracejada corresponde à luz branca emitida pelo LED, enquanto que as linhas vermelha e azul correspondem à mesma luz transmitida através de filtros de cor vermelho e azul. A luz branca emitida pela lanterna de LED possui eficiências de emissão diferenciadas nas regiões espectral do azul e do vermelho.

\section{Resultados e discussão}

Como flutuações temporais na intensidade da luz da fonte podem prejudicar os resultados do experimento para a avaliação da Lei de Malus, foi feita inicialmente uma avaliação da estabilidade de emissão da lanterna utilizando o software Power meter desenvolvido no trabalho. Para tanto, a luz da lanterna foi projetada diretamente sobre o anteparo e a intensidade relativa foi medida ao longo do tempo. Optou-se por utilizar uma fonte de tensão (Minipa, MPS-3503) para alimentar a lanterna. O resultado obtido pode ser visto na Fig. 4. Com o uso da fonte de tensão foi medida uma intensidade relativa média de $(47,39 \pm 0,15)$.

Em seguida foram realizados dois conjuntos de medições para a verificação da Lei de Malus utilizando a luz branca da lanterna de LED e a montagem experimental mostrada na 
Fig. 2a e 2b. Foram variadas as condições da iluminação ambiente de um conjunto de medições para o outro, mostrando os efeitos da luminosidade externa sobre o tempo de exposição da câmera e consequentemente na sensibilidade do detector. Os resultados são apresentados na Fig. 5. A mínima intensidade medida pelo detector é obtida com os polarizadores cruzados, o que equivale a um ângulo $\theta=90^{\circ}$ na expressão da Lei de Malus, equação (1). Os pontos experimentais correspondem ao valor médio de um conjunto de 30 medições realizadas em condições de repetibilidade. As barras de erros em y correspondem à incerteza combinada calculada a partir do desvio padrão do valor médio e da flutuação temporal da intensidade da luz do LED. As barras de erro em $x$ correspondem a um valor estimado de $\pm 0,5^{\circ}$ para a incerteza na determinação do ângulo. As medições consecutivas foram realizadas com um intervalo de $250 \mathrm{~ms}$. Aos pontos experimentais foram ajustadas funções cossenoidais ao quadrado, com um ângulo de fase inicial na fase da função harmônica. Essa fase inicial permite compensar qualquer inexatidão na determinação da posição angular associada à mínima intensidade transmitida (polarizadores cruzados).

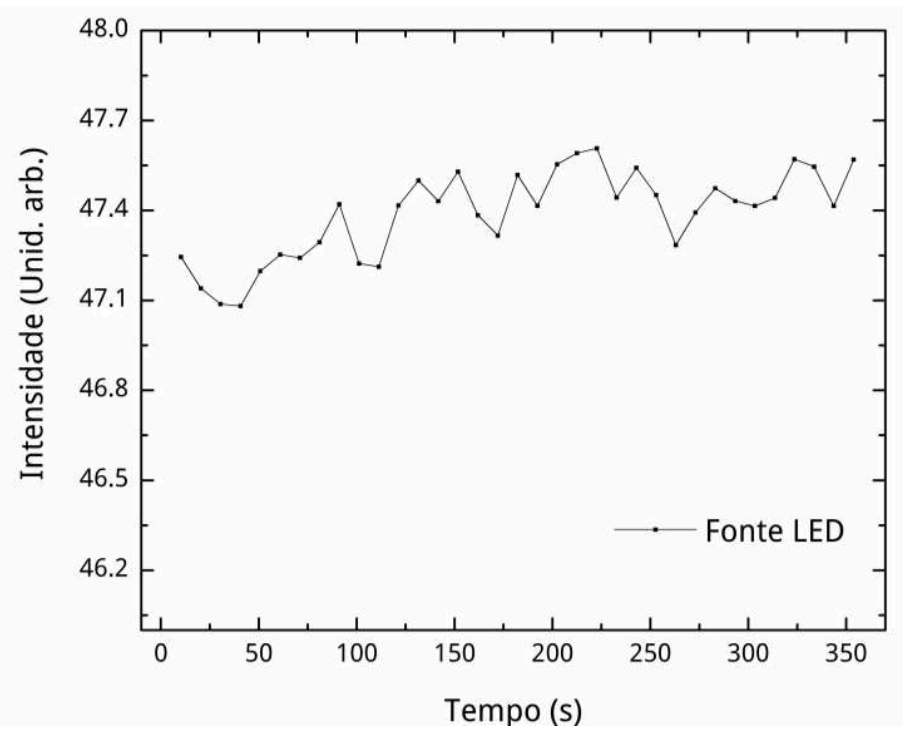

Fig. 4 - Gráfico temporal da intensidade relativa da luz emitida pela lanterna alimentada com a fonte de tensão externa.

Uma melhor visualização dos resultados obtidos com o experimento da Lei de Malus pode ser obtida pela linearização da equação (1), o que equivale à troca da grandeza representada no eixo $x$ do gráfico da Fig. 5, de $\theta$ para $\cos ^{2}(\theta)$. O resultado obtido pode ser visto no gráfico da Fig. 6, para a faixa de ângulos compreendida entre $90^{\circ}$ e $0^{\circ}$. Para ambos os casos, os coeficientes de correlação $R$ obtidos para o ajuste linear foram maiores do que 0,9988 . 


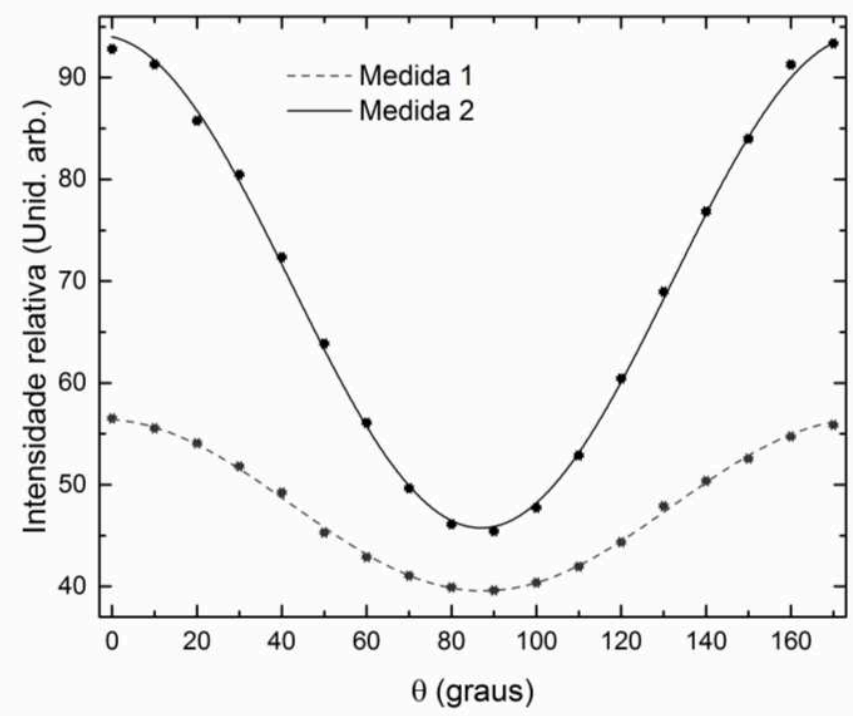

Fig. 5 - Gráfico da intensidade transmitida através de dois polarizadores, com um ângulo $\theta$ entre os seus eixos de polarização. As barras de erro em alguns casos são menores do que o tamanho do símbolo.

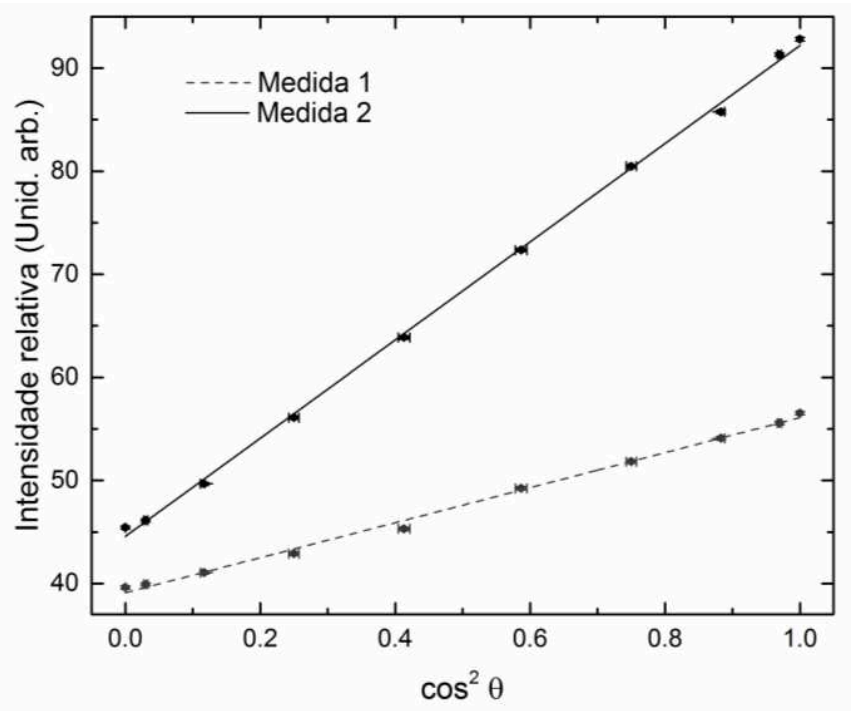

Fig. 6 - Ajuste dos dados experimentais à Lei de Malus na forma linearizada. As barras de erro em alguns casos são menores do que o tamanho do símbolo.

Mudanças nas condições de iluminação do ambiente alteraram os valores de intensidade relativa, porém não inviabilizaram o experimento.

O programa desenvolvido permite também analisar a eficiência dos polarizadores em diferentes regiões do espectro eletromagnético. A intensidade relativa versus ângulo entre 
polarizadores foi medida usando individualmente os filtros de cor vermelho e azul. No gráfico da Fig. 7 estão indicados os resultados obtidos com os dois filtros de cor.

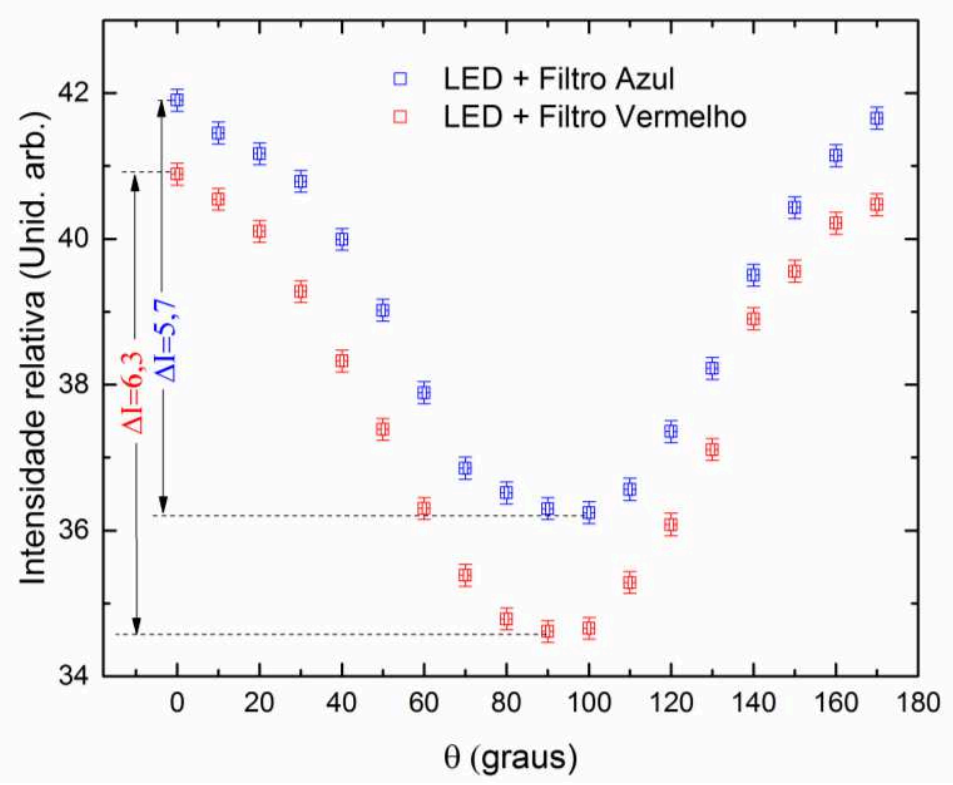

Fig. 7 - Comportamento da razão de extinção dos polarizadores, para as regiões espectrais do azul e do vermelho.

Cada ponto experimental do gráfico corresponde ao valor médio de 30 medições realizadas em condições de repetibilidade. As medições consecutivas foram realizadas com um intervalo de $250 \mathrm{~ms}$. As barras de erros em y levam em conta o desvio padrão do valor médio e a flutuação temporal da intensidade da luz do LED. As barras de erro em $x$ correspondem a incerteza de $\pm 0,5^{\circ}$ na determinação do ângulo.

Nos gráficos também está indicado o grau de polarização $\Delta I$ da luz após o analisador, definido na equação (3) (HECHT, 1987):

$$
\Delta I=\frac{I_{\max }-I_{\min }}{I_{\max }+I_{\min }}
$$

Nesta equação, $I_{\max } e I_{\min }$ representam as intensidades medidas com os polarizadores para $\theta=0^{\circ}$ e $\theta=90^{\circ}$, respectivamente. O menor grau de polarização obtido com o filtro azul $(\Delta I=5,7)$ quando comparado com o obtido com o filtro vermelho $(\Delta I=6,3)$ é resultado de uma maior fração não polarizada de luz azul que atravessa o conjunto polarizador-analisador, mesmo quando estes estão cruzados. A dependência entre o grau de polarização e o comprimento de onda da luz pode ser constatada qualitativamente olhando para uma lâmpada através de dois polarizadores cruzados. Nestas condições, observa-se que uma pequena fração da luz de tonalidade azulada atravessa os polarizadores. 


\section{Conclusões}

O sistema sensor proposto mostrou-se capaz de medir as mudanças de intensidade observadas em experimentos voltados para o estudo de fenômenos resultantes da polarização de ondas eletromagnéticas. Mostramos as aplicações do sistema em duas situações diferentes: na verificação experimental de Lei de Malus e na análise da eficiência de extinção dos polarizadores em diferentes regiões espectrais.

O programa desenvolvido utiliza uma câmera USB para leitura dos dados, podendo ser inclusive a própria câmera que vem instalada na maioria dos laptops, o que possibilita a realização de experimentos mesmo em laboratórios didáticos que não disponham de instrumentação adequada para medição de intensidade luminosa. O problema resultante da compensação automática da luz ambiente pela câmera foi resolvido estabelecendo uma região de referência de intensidade, próxima à região onde a medição é efetivamente realizada.

Adicionalmente, outros experimentos de polarimetria que envolvam medição de intensidade da luz podem ser realizados com o programa desenvolvido. Como exemplo, podem ser citados experimentos sobre atividade ótica natural de substâncias, ou experimentos de polarização por reflexão em interfaces dielétricas, na determinação dos coeficientes de Fresnel.

\section{Agradecimentos}

Os autores agradecem as agências de fomento Fundação Araucária, CAPES e CNPq.

\section{Referências}

COONEY, A. Aristochart. Line charting library. Disponível em: $<$ http://adriancooney.ie/aristochart>. Acesso em: 20 ago. 2016.

CATELLI, F.; VICENZI, S. Transformando um laser de diodo para experimentos de óptica física. Caderno Brasileiro de Ensino de Física, v. 21, n. especial, p. 319-331, 2004.

GITHUB INC. Electron. Library developed by GitHub for building cross-platform desktop applications. versão 1.4.1. Disponível em: <http://electron.atom.io>. Acesso em: 16 ago. 2016.

HECHT, E. Optics. 2. ed. Massachusetts: Addison Wesley, 1987. 676 p.

LAND, E. H. Some aspects of the development of sheet polarizers. Journal of the Optical Society of America, v. 41, n. 12, p. 957-963, 1951.

LOPES, É. M.; LABURÚ, C. E. Diâmetro de um fio de cabelo por difração (um experimento simples). Caderno Brasileiro de Ensino de Física, v.21, n. especial, p. 258-264, 2004. 
PEDROSO, L.S. et al. Construção de um luxímetro de baixo custo. Revista Brasileira de Ensino de Física, v. 38, n. 2, e2503, 2016.

RÄTY, J.A.; PEIPONEN, K.; ASAKURA, T. UV-visible reflection spectroscopy of liquid. 1. ed. Berlin Heidelberg: Springer-Verlag, 2004. 217p.

WALNES, J.; NOAKES, D. Smoothie charts. Charting Library for Streaming Data. Disponível em: <http://smoothiecharts.org>. Acesso em: 16 ago. 2016.

VERTCHENKO, L.; VERTCHENKO, L. Verification of Malus's Law using a LCD monitor and digital Photography. Revista Brasileira de Ensino de Física, v. 38, n. 3, e3311, 2016.

VIEIRA, L. P.; AGUIAR, C. E. Verificação da lei de Malus com um Smartphone. In: SIMPÓSIO NACIONAL DE ENSINO DE FÍSICA, XXI, 2015, Uberlândia. Anais... p. 1. 